\title{
Finite Element Modelling of Oxygen Diffusion and Segregation at Interfaces in Ag-MgO Composites: Parametric Studies
}

\author{
Andreas Öchsner ${ }^{1, \mathrm{a}}$, Irina V. Belova ${ }^{2, \mathrm{~b}}$, Graeme E. Murch ${ }^{2, \mathrm{c}}$ \\ ${ }^{1}$ Department of Applied Mechanics, Technical University of Malaysia, 81310 Skudai, Malaysia \\ ${ }^{2}$ Diffusion in Solids Group, Centre for Geotechnical and Materials Modelling, School of Engineering, \\ The University of Newcastle, Callaghan, NSW 2308, Australia
}

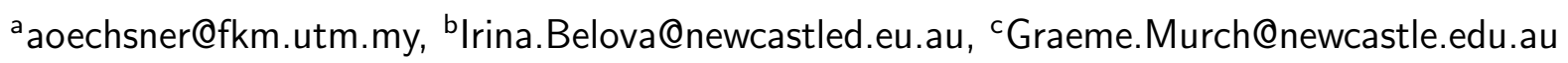

\section{Keywords: Segregation, Diffusion, Finite Element Method, Interfaces, Composites}

\begin{abstract}
The presence of atomic oxygen at internal metal-ceramic oxide interfaces significantly affects the physical properties of the interfaces which in turn affects the bulk properties of the material. This problem is addressed for the model system Ag-MgO from a phenomenological point of view using the finite element method. The performed parametric studies investigate the influence of different kinetic parameters of the diffusion-segregation system.
\end{abstract}

\section{Introduction}

Metal-ceramic phase boundaries are of great importance for many applications in materials science technology [1], e.g. to thin solid films, coatings, electronic packaging, supported catalysts, and fibre-reinforced metal-matrix composites. In many dispersion-hardened alloys (e.g. silver, copper, and nickel), metal-oxide interfaces are involved in strengthening mechanisms. They are also included in microminiature electronic devices, e.g. MOSFETs.

A useful technique to produce fine oxide particles uniformly dispersed in a metallic matrix is the internal oxidation of alloys [2]. Silver is one of the favourite base materials for internal oxidation experiments [3, 4]. This special status stems from practical reasons [5] since small volume fractions of an alloying addition (e.g. $\mathrm{Al}, \mathrm{Mg}, \ldots$ ), which forms the dispersed particles, result in significant hardening effects. The presence of solute atoms at internal metal-oxide interfaces influences the physical properties of the interfaces and this, in turn, may affect the bulk properties [6]. Therefore, it is an important task to accurately predict and measure the level of solute-atom segregation at internal interfaces. In papers $[7,8]$, the segregation of oxygen to metal-oxide interfaces has been numerically investigated based on one-dimensional finite difference models and the influence of various effects such as kinetic order or non-homogeneity of the oxide distributions were investigated. Later, more sophisticated geometries and particle distributions were investigated with a two-dimensional finite element scheme making use of commercial code that had been extended with special user-subroutines [9]. In recent papers $[10,11]$, the phenomenological problem of the time-dependent segregation of oxygen at metalceramic ( $\mathrm{Ag}-\mathrm{MgO})$ interfaces by making use of a lattice-based Monte Carlo method and a finite element method was addressed. In the present paper, the finite element method is applied to perform parametric studies to investigate the influence of different kinetic parameters of the diffusion-segregation system. The presented simulations are related to a state after the formation of oxide particles, i.e. the internal oxidation itself is not considered within the computational model. A schematic sketch of the physical problem is shown in Fig. 1. 


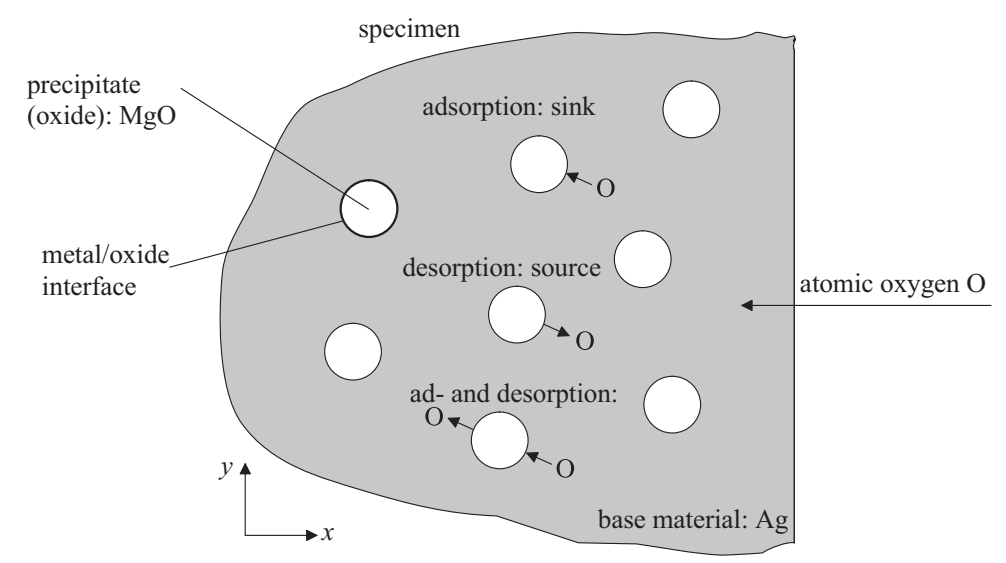

Fig. 1: Schematic description of the diffusion-segregation problem.

\section{Theory and Finite Element Modelling}

For the parametric studies, the same two-dimensional geometry and material parameters are used as described in [10]. Oxide particles are modelled as square inclusions which are arranged in a square planar pattern, cf. Fig. 2 a. At the right hand side of the model, a constant concentration $\psi_{\mathrm{O}}^{1}=1$ is prescribed as the boundary condition and the left hand side is assumed to extend to infinity. The infinite boundary is realised by consideration of sufficient unit cells so that the initial conditions $\left(\psi_{\mathrm{O}}^{1}=0\right)$ are still fulfiled at this boundary. This conditions has been checked for each single simulation.

a)

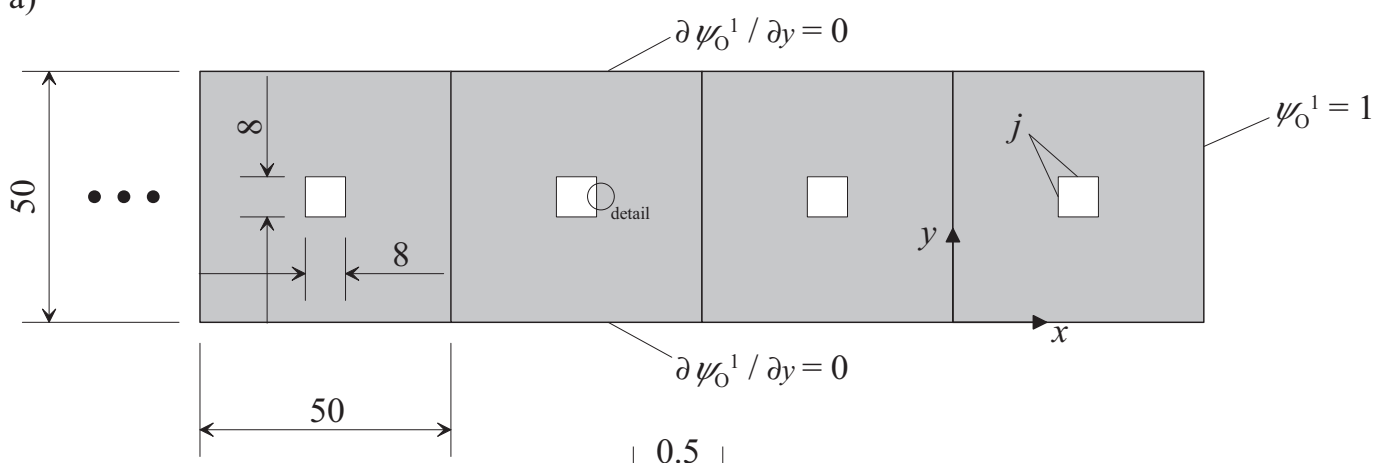

b) mesh detail

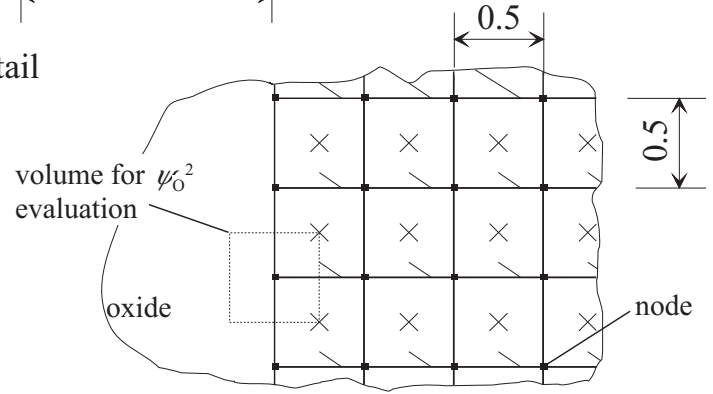

Fig. 2: a) Schematic representation of the modelling approach for the adsorption/desorption (segregation) of oxygen shown in Fig. 1; b) details of the finite element mesh.

The mesh itself (cf. Fig. 2 b) is composed of equal four-node, isoparametric square elements with bilinear interpolation functions. A single unit cell $(50 \times 50)$ comprises 9744 elements of dimension $0.5 \times 0.5$. It should be noted here that only the base material is represented by finite 
elements and the oxide particles are represented by empty cutouts in the middle of each unit cell.

The kinetics of oxygen diffusion in the base material can be described based on Fick's second law of diffusion for the oxygen concentration $\psi_{\mathrm{O}}^{1}$ as

$$
\frac{\partial \psi_{\mathrm{O}}^{1}}{\partial t}=D_{\mathrm{O}}^{1} \Delta \psi_{\mathrm{O}}^{1}
$$

where $D_{\mathrm{O}}^{1}$ denotes the diffusion coefficient of oxygen in the alloy and $\Delta=\left(\frac{\partial^{2}}{\partial x^{2}}+\frac{\partial^{2}}{\partial y^{2}}\right)$ is the Laplace operator. The segregation of the oxygen atoms at the metal/oxide interfaces is modelled as a boundary flux

$$
q=-w_{12} \cdot \psi_{\mathrm{O}}^{1}+w_{21} \cdot \psi_{\mathrm{O}}^{2},
$$

where $w_{12}$ and $w_{21}$ are local transition rates which relate to the equilibrium segregation factor $s=w_{12} / w_{21}$. The oxygen concentration $\psi_{\mathrm{O}}^{2}$ at the surface of the oxide particles is obtained by integration of the area-related flux and relating this value to the correspondent volume $\Delta V=0.5 \cdot 0.5 \cdot c$ (thickness $c=1$ ), cf. Fig. 2 b:

$$
\psi_{\mathrm{O}}^{2}=\Sigma q_{i} \cdot \frac{\Delta A}{\Delta V} \cdot \Delta t .
$$

In Eq. (3), the area $\Delta A$ which is perpendicular to the boundary flux is obtained as $\Delta A=0.5 \cdot c$ and $\Delta t=1$ is the time increment of the solution procedure.

\section{Results}

The first example illustrates the influence of different local transition rates $w_{12}$ on the oxygen concentration profiles. Figure 3 shows that higher transition rates (which is equal to a stronger adsorption) results in lower concentration profiles on both sides of the oxide particle.
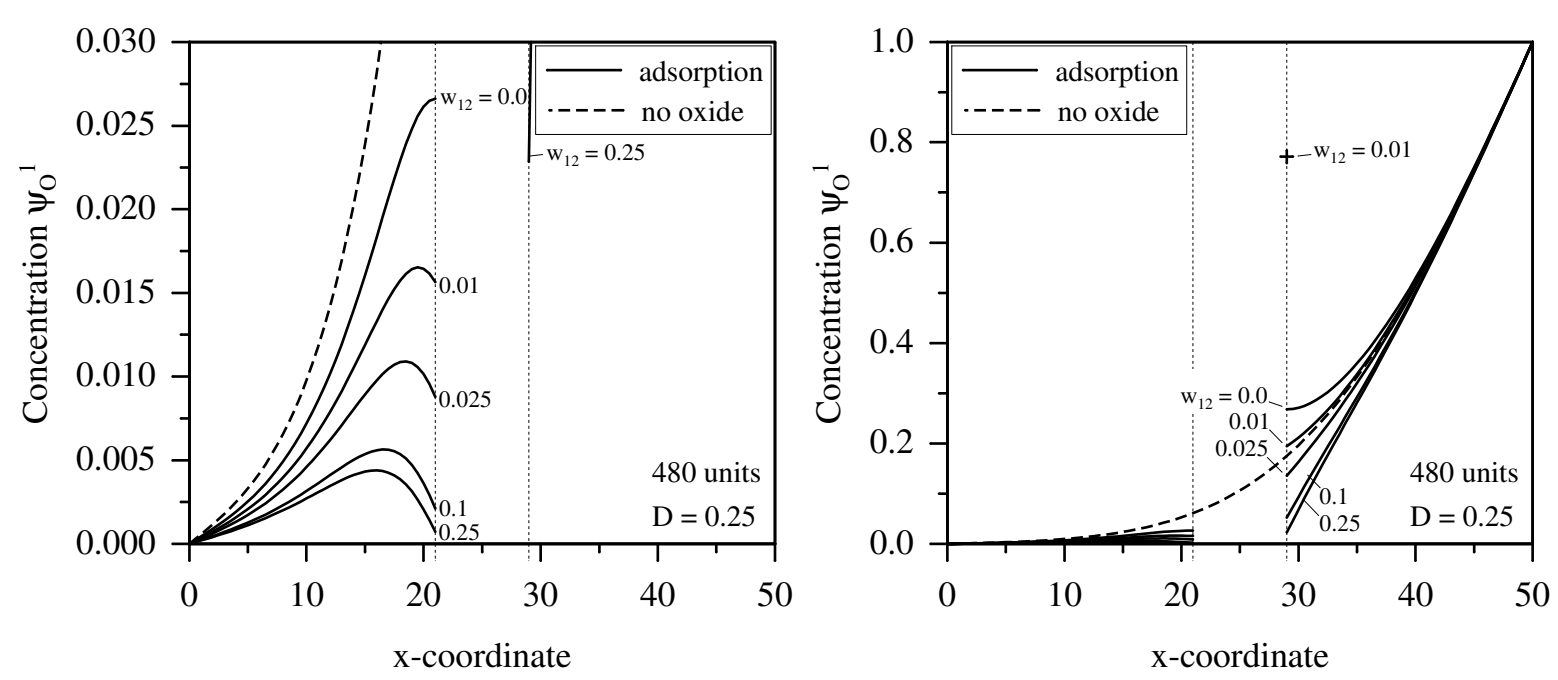

Fig. 3: Magnification of oxygen concentration profiles for different transition rates $w_{12}$ along $y=25$.

The dashed line in this figure refers to a simulation where no oxide particle is present. This has been achieved by meshing the empty space of the oxides and assigned the same properties 
as in the base material. A clear trend, i.e. that the concentration is higher in this special case, can be only observed for the part of the base material which is in the 'shadow' of the oxide particle.

Figure 4 illustrates the dependency of the oxide particle concentration on different transition rates. Only on the right hand side of the particle, i.e. where the base material faces the free boundary, a monotonically behaviour of the point values can be observed: higher values of the transition rate $w_{12}$ reveal a higher point value $\psi_{O}^{2}$. The situation on the left hand side is not very clear since the point values have no monotonically trend.
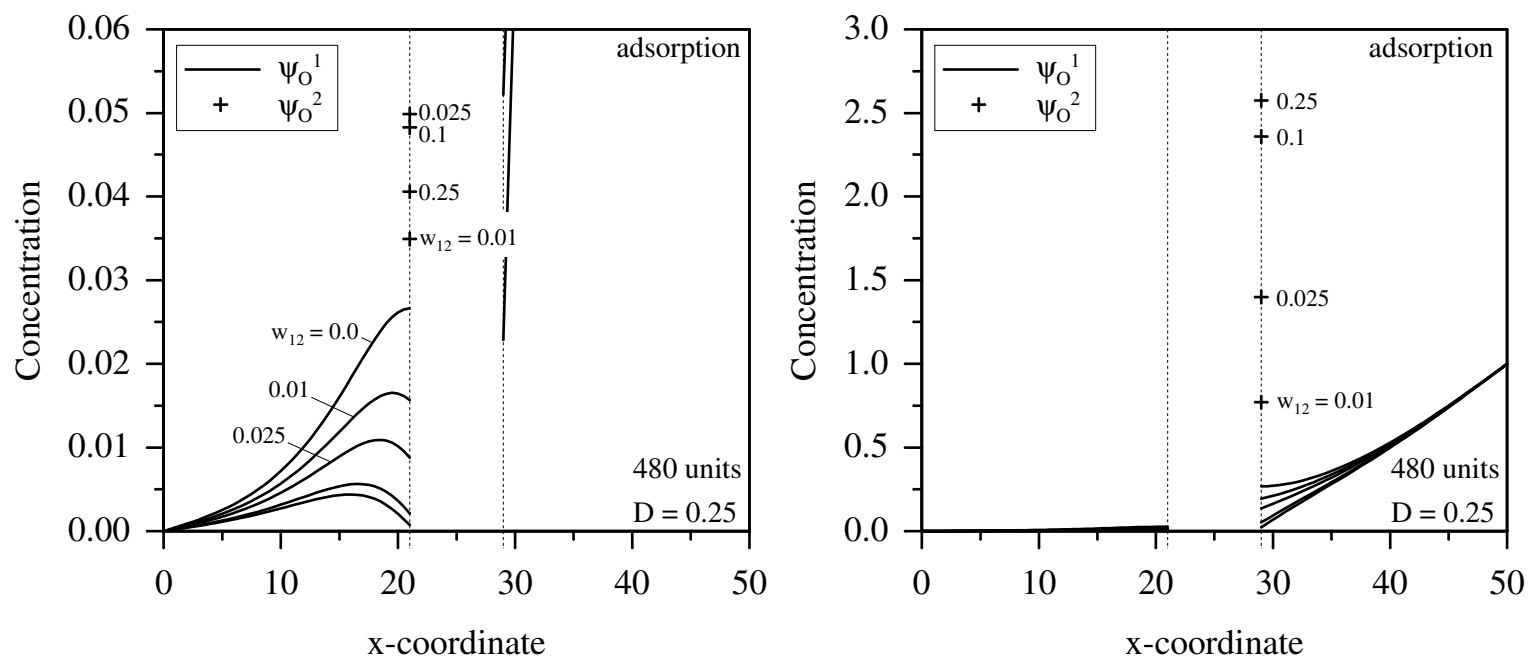

Fig. 4: Oxygen concentration profiles $\left(\psi_{\mathrm{O}}^{1}\right)$ for different transition rates $w_{12}$ along $y=25$ and point values of $\psi_{\mathrm{O}}^{2}$ at $(x=21, y=25)$.

In order to investigate this behaviour, Fig. 5 presents the concentration and flux values as a function of time. It can bee seen that the concentration behaves monotonically but that the boundary flux on the left side of the particle reveals the same non-monotonically trend for different values of the transition rate in this region.
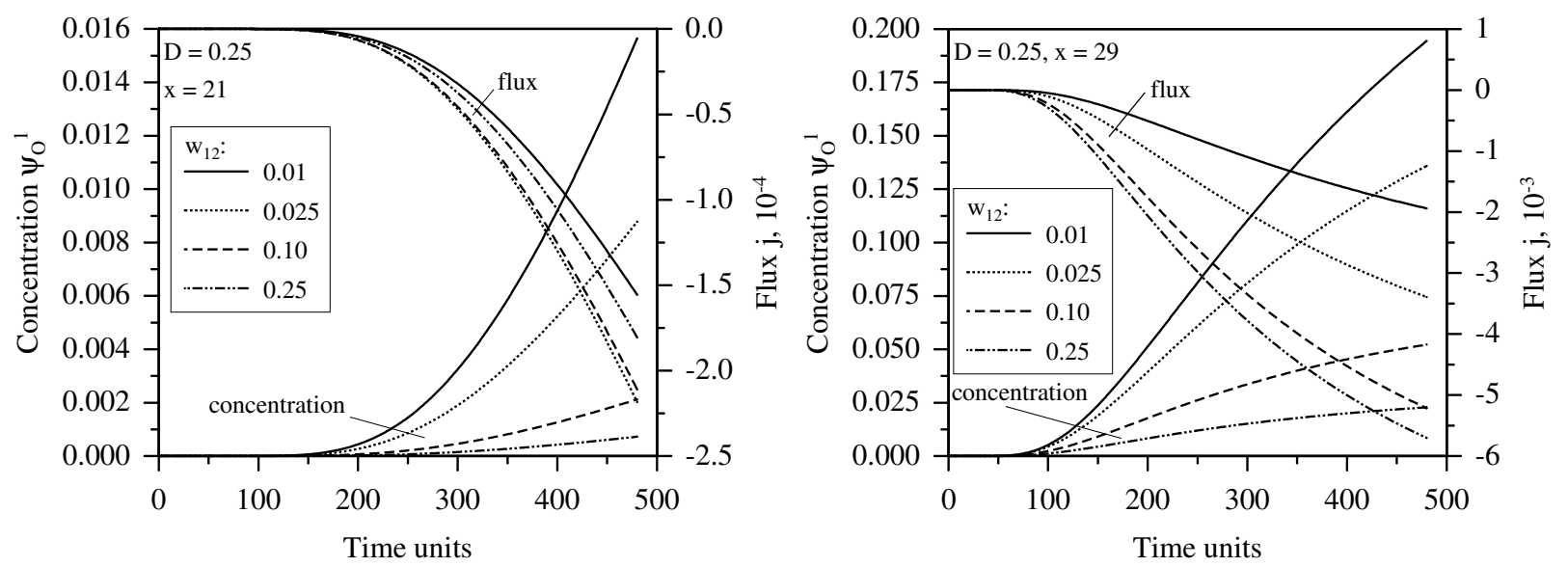

Fig. 5: Evaluation of concentration and boundary flux with time at $y=25$ and $x=21$ (left) and $x=29$ (right). 
In contrast to this behaviour, flux and concentration increases on the right hand side with decreasing $w_{12}$. It must be concluded that the situation in the 'shadow' of the particle is quite complex and that different effects (such as shielding due to the particle, boundary value of concentration and flux) are competing. Figure 7 summarises this complex behaviour in the 'shadow' of the oxide particle. As can be seen from this figure where a steep monotone increase up to a maximum if followed by a monotone decrease, the point concentration reveals a nonmonotonic dependency on the transition rate $w_{12}$.

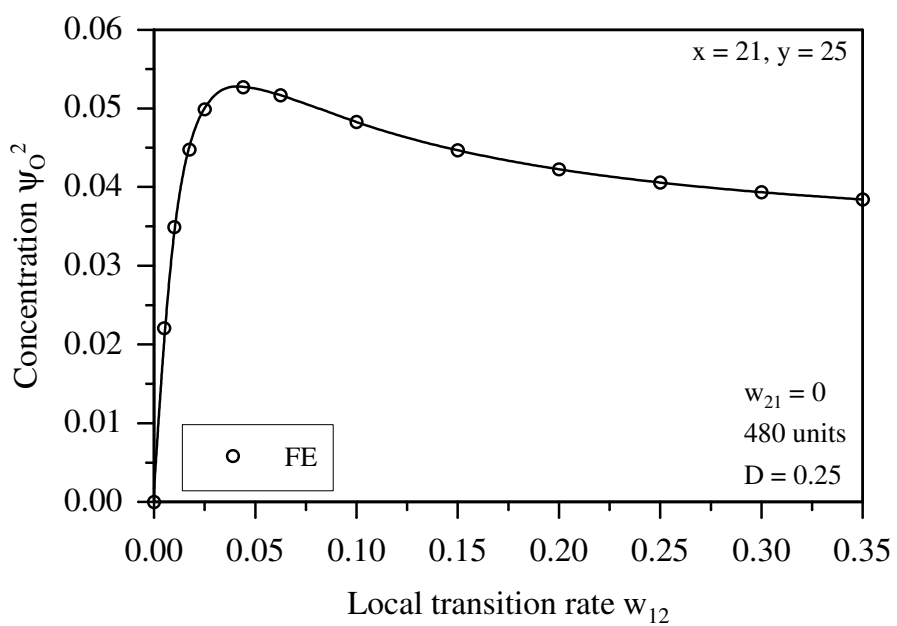

Fig. 6: Evaluation of point flux values of $\psi_{\mathrm{O}}^{2}$ for different transition rates $w_{12}$.

The last example illustrates the influence of the desorption on the concentration profiles, cf. Fig. 7. If the time is small (480 units), no difference between the simulation with pure adsorption and ad- and desorption can be observed under the chosen model parameters.

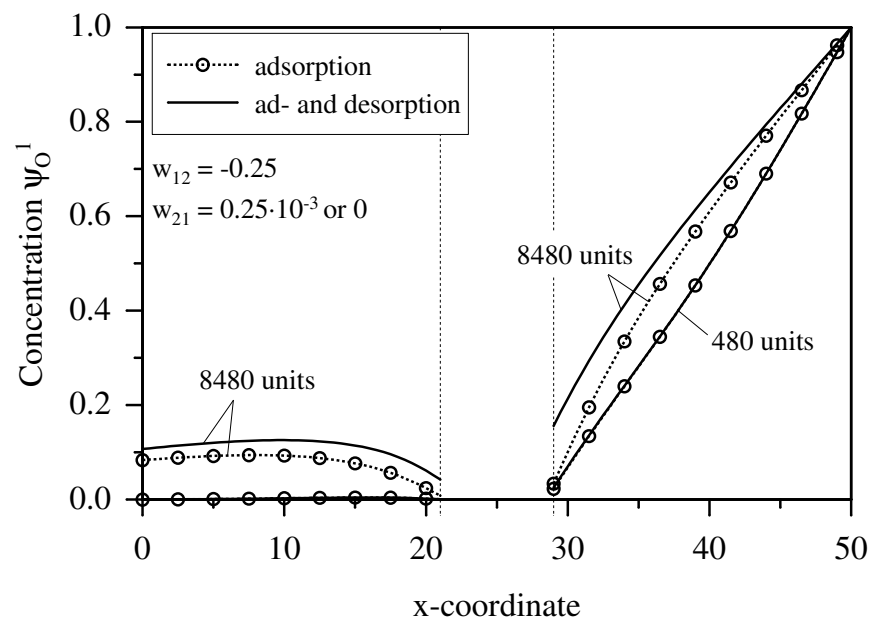

Fig. 7: Oxygen concentration profiles $\left(\psi_{\mathrm{O}}^{1}\right)$ for different time steps.

Larger times (8480 units) result in a visible difference between both simulation. The simulation only based on adsorption underestimates the values obtained by consideration of adsorption and desorption. This conclusion is valid for both sides of the oxide particle. A comparison of the numerical values of the deviation between both simulation (cf. Tab. 1) indicates that the 
error is for a given time step more of less of the same magnitude and clearly increasing for longer time steps.

\begin{tabular}{ccrrr} 
time (units) & $x$ (comp.) & $\psi_{\mathrm{O}}^{2}$ (adsorption) & $\psi_{\mathrm{O}}^{2}$ (ad- and desorption) & deviation $(\%)$ \\
\hline \multirow{2}{*}{480} & 21 & 0.040562 & 0.040880 & -0.777517 \\
\multirow{2}{*}{2173} & 29 & 2.575804 & 2.559939 & 0.619735 \\
& 21 & 3.429381 & 3.550025 & -3.398384 \\
\multirow{2}{*}{8480} & 29 & 28.681322 & 28.140822 & 1.920699 \\
& 21 & 28.113477 & 32.050623 & -12.284147 \\
& 29 & 134.927429 & 126.503445 & 6.659094
\end{tabular}

Table 1: Comparison of the point values of $\psi_{\mathrm{O}}^{2}$ at $y=25$ obtained by finite element method.

\section{Outlook}

It could be shown that different kinetic parameters of the diffusion-segregation system can significantly influence the oxygen concentration profiles. The situation in the 'shadow' of an oxide particle is quite complex and cannot be predicted without a detailed numerical simulation. Another interesting process is the out-diffusion of oxygen which is performed experimentally to remove oxygen from the composite including the interphase layer. This reverse diffusion process is now being addressed with finite element and Lattice Monte Carlo simulations by the present authors. The cited work based on the lattice-based Monte Carlo method [10, 11] and the here applied finite element method are powerful tools to simulate the oxygen diffusion and segregation at interfaces in metal-ceramic composites. The next step must be to simulate how this segregation influences for example the macroscopic mechanical properties. To this end, very sophisticated investigations of the metal-ceramic oxide interface will be required.

\section{Acknowledgements}

Financial support of the Australian Research Council is gratefully acknowledged.

\section{References}

[1] A. Gonis and P. Tuchi: Stability of Materials. Structure-Property Relationship of Metal-Ceramic Interfaces (Gordon \& Breach, USA 1996).

[2] J. Gegner and A. Weible: Z. Metallk. Vol. 91 (2000), p. 1026

[3] R.A. Rapp: Acta Met. Vol. 9 (1961), p. 730

[4] V.A. van Rooijen, E.W. van Royen, J. Vrijen and S. Radelaar: Acta Met. Vol. 23 (1975), p. 987

[5] D. Stöckel and H.J. Grabke: Z. Metallk. Vol. 64/4 (1973), p. 287

[6] D. Wolf and S. Yip: Materials Interfaces (Chapman \& Hall, UK 1992).

[7] M. Stasiek, A. Öchsner and J. Grácio, in: Proceedings of the 1st International Conference on Diffusion in Solid and Liquids DSL-2005, A. Öchsner, J. Grácio and F. Barlat, July 6-8, 2005, University of Aveiro, Aveiro, Portugal. p 723-726

[8] A. Öchsner, M. Stasiek and J. Grácio: Defect Diffus. Forum Vol. 249 (2006), p. 35

[9] Stasiek, A. Öchsner and J. Grácio: J. Phase Equilib. Diff. Vol. 27 (2006), p. 644

[10] I.V. Belova, G.E. Murch, N. Muthubandara and A. Öchsner: Solid State Phenom. Vol. 129 (2007), p. 111

[11] I.V. Belova, G.E. Murch and A. Öchsner: submitted to Defect Diffus. Forum (2007) 